\title{
Patients with clinically metabolically healthy obesity are not necessarily healthy subclinically: further support for bariatric surgery in patients without metabolic disease?
}

\begin{abstract}
Background: Nonalcoholic fatty liver disease (NAFLD) increases the risk of liver cirrhosis and hepatocellular carcinoma and is also strongly correlated with extrahepatic diseases, including cardiovascular disease and type 2 diabetes. This risk of NAFLD among obese individuals who are otherwise metabolically healthy is not well characterized. Objectives: To determine the prevalence and characteristics of NAFLD in individuals with metabolically healthy obesity. Setting: A tertiary, academic, referral hospital. Methods: All patients who underwent bariatric surgery with intraoperative liver biopsy from 2008 to 2015 were identified. Patients with preoperative hypertension, dyslipidemia, or prediabetes/diabetes were excluded to identify a cohort of metabolically healthy obesity patients. Liver biopsy reports were reviewed to determine the prevalence of NAFLD. Results: A total of 270 patients $(7.0 \%$ of the total bariatric surgery patients) met the strict inclusion criteria for metabolically healthy obesity. The average age was $38 \pm 10$ years and the average body mass index was $47 \pm 7 \mathrm{~kg} / \mathrm{m} 2$. Abnormal alanine aminotransferase $(>45 \mathrm{U} / \mathrm{L})$ and asparate aminotransferase levels (>40 U/L) were observed in $28(10.4 \%)$ and $18(6.7 \%)$ patients, respectively. A total of 96 (35.5\%) patients had NAFLD with NALFD Activity Scores 0 to 2 $(\mathrm{n}=61), 3$ to $4(\mathrm{n}=25)$, and 5 to $8(\mathrm{n}=10)$. A total of $62(23 \%)$ patients had lobular inflammation, $23(8.5 \%)$ had hepatocyte ballooning, 22 (8.2\%) had steatohepatitis, and 12 (4.4\%) had liver fibrosis. Conclusion: Even with the use of strict criteria to eliminate all patients with any metabolic problems, a significant proportion of metabolically healthy patients had unsuspected NAFLD. The need and clinical utility of routine screening of obese patients for fatty liver disease and the role of bariatric surgery in the management of NAFLD warrants further investigation.
\end{abstract}

Keyword: Bariatric surgery; Liver disease; Metabolically healthy obesity; Metabolic syndrome; Nonalcoholic fatty liver disease; Diabetes; Insulin resistance; Steatohepatitis 